\section{Mel 05}

FLOW CYTOMEIRIC (FCM) STUDIES ON THE BIOLOGY OF MALIGNANT MELANOMAS AND PREMALIGNANT LESIONS J. Schumann, I. Suter and W. Göhde

FCM DNA-measurements were done in 434 primary nital melanocytic nevi. Abnormal DNA-stemlines were detected in $41 \%$ of the primary tumors, in $75 \%$ of the metastases and in $8 \%$ of the congenital nevi. The occurrence of abnormal DNA-stemlines proved to be an indicator of poor prognothus denoting tumor cell heterogeneity. course of the disease in 105 patients. Genetic instability - that means changes of the DNA-invalues - was observed in $62 \%$ of the patients evaluated. The course of the disease in these cases was more progressive, resulting in a shorter survival time. Compared with tumor cell heterogeneity, genetic instability seems to be a superior prognostic criterion indicating poor WELL's hypothesis (Science, 194, $23-28,1976$ ) that genetic instability may select more profressive cell lines.

The occurrence of abnormal DNA-cell lines in congenital melanocytic nevi probably is an indicator of a premalignant condition in cases where histologic examination does not reveal any evidence for malignant melanoma.

Fachklinik Hornheide - University of Münster 4400 Münster, FRG melanomas, 480 melanoma metastases and 59 congesis. $37 \%$ of the primary tumors were multiclonal Sequential measurements were possible during the dex in the tumor cell lines to higher or lower prognosis. These results obviously confirm NO-

\section{Mel 07}

CHARACTERIZATION OF CELL SURFACE ANTIGENS DISTINGUISHING BENIGN AND MALIGNANT HUMAN MELANOCYTES

B. Holzmann, J.P. Johnson and G. Riethmüller

A considerable number of monoclonal antibodies directed against melanoma associated antigens have been described. However, most of these antigens were found to be expressed on benign melanocytic lesions in essentially the same way as on melanoma cells. Recently we have described two glycoproteins, gp75 and gp 89, which are differentially expressed on benign and malignant melanocytic lesions.

In a more extended study 42 benign nevi and 56 malignant melanomas were investigated by immunohistochemical methods. Whereas $76 \%$ of the melanomas expressed gp75 and $89 \%$ gp 89 , only $2 \%$ of the nevi were positive for gp 75 and $14 \%$ for gp 89. The expression of both antigens was not homogeneous within a given lesion but revealed distinct subpopulations. Staining of nevi was usually weaker than melanomas and less cells were labelled.

For further investigation of these antigens, molecular cloning of gp89 was approached by DNA transfection into mouse L-cells. Clones from the second round of transfection were obtained which express gp 89. Attempts to isolate the gene for gp 89 by hybridization with human DNA probes are in progress.

In addition, structural variation of gp 89 observed in different cell lines is being further investigated.

Institute of Immunology, University of Munich, Goethestr. 31, D-8000 München 2, F.R.G.

Mel 08

SENSITIVITY, SELECTIVITY, AND SPECIFICITY OF MONOCLONAL ANTIBODIES DIRECTED AT TUMOR ASSOCIATED ANTIGENS FROM METASTATIC MELANOMA

U.R.Kleeberg, A. Loycke, T. Meier, E. Bröcker, F. Lejeune, M. Prade, S. Carre]

for the EORTC Malignant Melanoma Coop. Group (MAB-RDP)

of cytotoxic $T$ cells with target cells. Class II HLA (DR, DP, DQ) antigens that are constitutively expressed on antigen-presenting cells can be induced in a variety of cells by $y$ interferon.

Normal melanocytes bear low amounts of class I and lack class II HLA antigens. Immunohistological studies on benign and malignant melanocytic tumors with monoclonal antibodies against class I and II HLA antigens revealed that HLA $A B C$ was absent or low in dermal nevi, and strongly expressed by nevi with mononuclear cell infiltrates. Nevi, however, lacked considerable amounts of HLA-DR, DP and $D Q$ antigens. Primary melanomas broadly expressed HLA-ABC antigens in most cases. Considerable expression of class II thlA was found in $40 \%$ of primary melanomas, and increased in proportion to invasiveness of the tumors. HTA-DR (and DQ) expression in primary melanoma was significantly associated with the amount of intratumoral $T$ cell infiltrates. The peritumoral infiltrate was not related to the class II phenotype of the tumors. Independently from tumor thickness, expression of HLA-DR on more than $10 \%$ of tumor cells was significantly associated with metastatic spread of primary tumors.

In melanoma metastases, we found a lack of HLA-ABC in advanced stages (visceral metastases). This feature, when present in locoregional metastases, was an adverse prognostic sign. Even worse, however, was broad expression of HLA-DR antigens in locoregional metastases. Metastases expressed more of ten HLA-DR and $-D P$ antigens than primary tumors.

our data suggest that loss of class I HLA-antigens. and increased expression of class II antigens are associated with poor prognosis of melanoma.

1 Dept.Dermatology, University of Münster, West Germany 2Dept. Pathology, University of Leiden, The Netherlands 3 Fachklinik Hornheide, Münster-Handorf, West Germany
Melanoma as any other cancer develops biological heterogeneity from a single clone origin. This heterogeneity manifests itself as a phenotypic variation with alteration of cellular morphology, varying sensitivity to immune defences, varying growth rates, variable metastatic potential including organotropy and varying susceptibility to therapeutic interventions.

Members of the EORTC-MMCG have recently developed a number of $M A B$ directed against various melanoma associated antigens using a common bank of 140 melanoma cell 1 ines. 30 purified antibodies were testes within the group and the most efficient selected for further development.

Helanoma metastases from regional 1 ymphnodes and various organs, bone marrow, normal human and metastatic tissue and from a variety of ectodermal, entodermal, and mesenchymal tumors were examined. Imprint as well as aspiration cytology was prepared before freezing the material and preparing cryostat sections.

The results show a wide variety of interesting crossreactions against normal and neoplastic human tissue, particularly with neurectodermal differentiation antigens and ontogenetically similar tumors.

It appears possible to reproducibly characterize the quality and sensitivity of $M A B$ directed against melanoma associated, which is a conditio sine qua non for their use in clinical medicine. It has become possible to use a panel of MAB-conjugated markers for in vivo and in vitro tumor imaging as well as conjugated cytostatic agents for a specific therapy.

Haemat.-onk. Praxis Altona, 2000 Hamburg 50, Germany Secretariat of the EORTC Malignant Melanoma Coop. Group 